(C)2007 IEEE. Personal use of this material is permitted. However, permission to reprint/republish this material for advertising or promotional purposes or for creating new collective works for resale or redistribution to servers or lists, or to reuse any copyrighted component of this work in other works must be obtained from the IEEE 


\title{
A Method for Service Quality Assessment in a Service Ecosystem
}

\author{
Chen Wu, Student Member, IEEE, ELIZABETH CHANG, Member, IEEE \\ Chen Wu and Elizabeth Chang, School of Information Systems, Curtin University of Technology, Australia, e-mail : \\ \{Chen.Wu, Elizabeth.Chang\}@cbs.curtin.edu.au
}

\begin{abstract}
- the increasing proliferation of independent application service providers is making traditional IT infrastructure insufficient when dealing with new issues that appear in a dynamic outsourcing business model. Quality of Service (QoS) gradually becomes an essential benchmark to differentiate diverse service providers during service selection process. In this paper, we argue service selection can be deemed as a decision making process - to decide which services providers should be selected within the specified service provision context during a definitive timeslot. Thus, existing decision support approaches can be leveraged if applicable. Hence, we propose a service selection solution which utilizes the Decision Support Systems Module (DSS Module) to select the most appropriate service. In DSS module we introduce AHP model to carry out the service QoS measurement based on the Context-specific Quality Aspects. The contributions of this paper are two folds. Firstly, we provide a novel and feasible solution for QoS-based service selection and secondly, we apply DSS module into web services, thus opening a new, fertile ground for DSS research in service ecosystem literature.
\end{abstract}

Index Terms-QoS, service selection, service ecosystem

\section{INTRODUCTION}

The significance of Quality of Services (QOS) in the performance of the web is evident. In a Service Ecosystem[1], where services are discovered, bound, and consumed in a dynamic loosely-coupled manner, it becomes particular important to specify, track, and manage QoS in order to select the most appropriate service providers. As a large number of enterprises start to adopt the emerging services paradigm to facilitate various cross-organisational business behaviours - e.g. the outsourcing of marginal functions [2], a reliable and empirical QoS solutions are essential to support such business routines in the SOE.

Recently, Digital Ecosystem has become the hosting environment for the services. In our previous work[1], we proposed a service ecosystem architecture and we have found the well-known issue of service selection [3] can be effectively tackled by employing certain DSS solutions for the following two reasons. Firstly, existing web services standards failed to provide a flexible, dynamic, and reliable mechanism to allow service requestors to choose the right quality service instance based on non-functional attributes such as QoS and trust. Secondly, in service-oriented environments, service selection can be seen as a process of decision making, i.e. the service requestor should make a decision on which service provider is currently offering the most appropriate service to fulfil the requirement among abundant function-relevant service provider candidates. This ar- gument is partly based on the assumption that DSS 'support' rather than 'automated' decision making [4], a task which cannot be completely achieved without human participation and final decision making. Hence, while some research attempts to automate the service selection without human intervention, it is our belief that such a thorough automation will not occur in the reality due to the complexity of service selection. Consequently, in this paper, we mainly deal with the issue of how to support the decision making process with regards to selecting the most appropriate services providers using DSS modules. We integrate into existing distributed web services architecture a DSS module which would assess the QoS of web services based on the Analytic Hierarchy Process (AHP) modelling approach. An application based on such a conceptualization is provided.

\section{Preliminary CONCEPTS}

Before we discuss the module design and architecture we would like to introduce some preliminary concepts on QoS assessment, which would facilitate understanding of our work in service selection DSS in Service-Oriented Environment.

\section{A. QoS Assessment within Service Context}

According to [5], QoS assessment can be carried out based on the concept of Service Context. A Service Context defines the "nature of the service and service functions". Each context has a name, a type and a functional specification. In this paper, we believe one can further derive from the nature of the service a set of Context Elements that constitutes the whole Service Context. For example, as illustrated in Figure 1, three context elements - functional features, QoS, and trust - are derived from the overall service context. These proposed context elements are considered playing a significant role in selecting the service providers as they characterise the nature of the service from different perspectives. Consequently, in most cases, all these elements shall be captured and assessed during the service selection process. Hence the process of selecting services, namely the decision making, is in effect the process of assessing these three elements respectively. For each Context Element, a service requester may employ different approaches to measure and assess. In this paper, we mainly deal with QoS assessment. In particular, we utilises the decision support module (i.e. the AHP modelling approach) to assess the QoS and, as a result, to select the most appropri- 
ate service providers. It is worth noting that future work is considered highly essential to assess other Context Elements under such a context-based framework. In addition, it is also an interesting topic to explore all the possible Context Elements that reveals the nature of the services in various business domains.

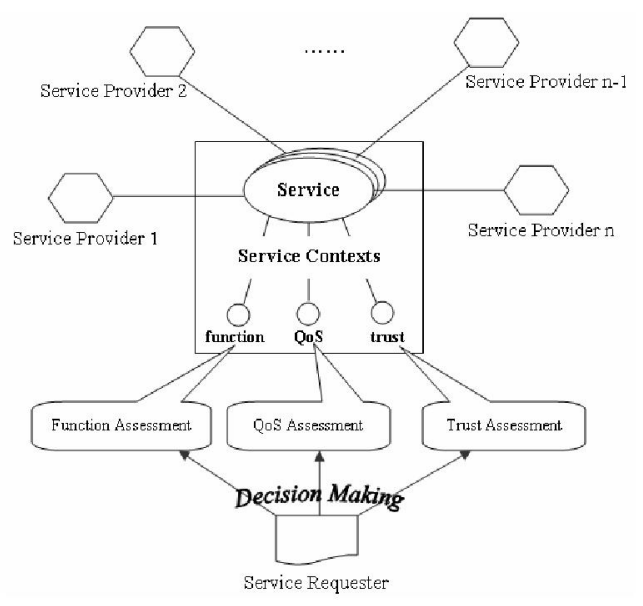

Figure 1. QoS Assessment in Service Context

\section{B. Quality Aspects for QoS Assessment}

When the Context Element QoS is studied separately, the Service Context can be broken down into a set of mutuallyindependent Quality Aspects (QA), each of which reflects one dimension in terms of the contribution towards the final overall QoS [5]. In other words, Quality Aspects decompose the Service Context into several dimensions for the purpose of quality assessment or measurement. Moreover, for each QA, there always exists a set of Assessment Criteria $(\mathrm{AC})$ that can be used to measure the quality within that particular context aspect. Assessment Criteria define the quality metrics for each QA of the Context for the purpose of measuring the delivered quality (aspects) against the defined quality (criteria). Consequently we believe that a hierarchical structure (See Figure 2) is essential for modelling such a decision making process - to select the most appropriate service providers based on all the hierarchically organised assessment criteria. To illustrate Figure 2 with an example, let us consider such a Service Context: 'A cargo delivery service provided by a third party logistics company located in the Western Australia'. The QoS Context Element of this Service Context can be further decomposed into four Quality Aspects: intact delivery, on-time service, tracking \& tracing capability, and money back guarantee. It can be noted that Quality Aspects with their associated Assessment Criteria could be defined by existing business (technical) standards, or derived from the established Service Level Agreement (SLA), or even generated at run-time via bilateral negotiation. Under each Quality Aspect in Figure 2, we can see several subordinate 'Assessment Criteria' associated with them. To continue with the previous example, for the QA on-time service, we can set up two Assessment Criteria: 1) on-time pickup, and 2) on-time delivery. Note that both ACs are considered during their quality as- sessment, nevertheless, they might have different contributing weight in views of different stakeholders. We will address such a criteria weight issue in Section III.

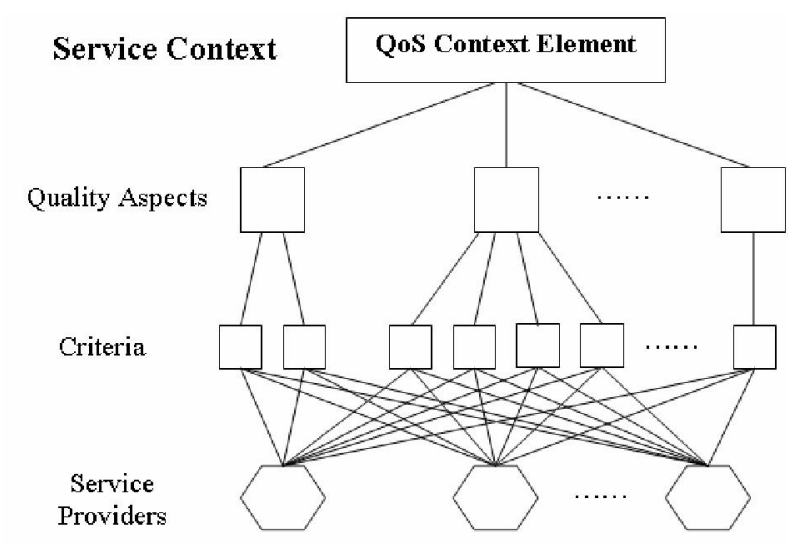

Figure 2. Hierarchical Structure of Quality Aspects

\section{AHP Modelling}

The Analytic Hierarchy Process (AHP), originally proposed in Saaty [6], allows decision makers to model a complex problem in a hierarchical structure showing the relationships of the goal, factors, sub-factors, and alternatives. The most salient characteristic of AHP is that it enables decision-makers to derive ratio scale priorities or weights (these two words are used interchangeably in this section) as opposed to arbitrary assigning them the absolute values. In doing so, AHP not only enables decision-makers to structure complexity and exercise judgment, but allows them to incorporate both objective and subjective considerations in the decision process [7]. More importantly, unlike other decision-aiding methods, AHP relies on relative weighting (pair-wise comparison) and is thus less susceptible to judgment errors common to other methods using absolute assignments [8]. Hence, in order to solve a complex problem with a hierarchical structure as indicated in Section 2.2, it is desirable to employ the AHP approach for the purpose of QoS assessment. In particular, we have found the corresponding relations between the concepts in AHP and the ones in QoS assessment as shown in Table 1 below. It is not difficult to notice from Table 1 that the QoS assessment research problem matches the original AHP model very well except the concept Stakeholder, which is thoroughly dealt with in Section III.

Table 1. Correspondence between AHP and QoS

\begin{tabular}{|c|c|}
\hline Concepts in AHP & Concepts in QoS \\
\hline Goal & QoS Assessment \\
\hline Factors & Quality Aspects \\
\hline Sub-Factors & Assessment Criteria \\
\hline Alternatives & Service Providers \\
\hline Decision makers & Stakeholders \\
\hline
\end{tabular}

\section{THE AHP APPROACH}

In this section, we propose an AHP-based modelling ap- 
proach to compute the alternative service providers' QoS value scores that are evaluated across a set of QA associated with the service context. Such an approach can be illustrated in Figure 3. It consists of the following major six steps.

\section{A. Original Data Collection}

The first step prepares the input for constructing the AHP hierarchical structure with the QoS concepts. According to the concepts matching relationship in Table 1 , the AHP approach in this research requires three primary inputs (as indicated by the grey arrows in Figure 3):

1. Quality Aspects with all their associated Assessment Criteria. As mentioned earlier, this information can be obtained from existing standards, SLA, or run-time contracts in the form of XML schema.

2. Candidate service providers' relative QoS ratio value against QAs and ACs that has been acknowledged in the first input. No absolute values are assigned here, rather, only the pair-wise comparison values are computed and evaluated.

3. Preference (or weight) on QA and AC set by different stakeholders. For example, the sales and trading department may be more concerned about 'on-time service' provided by the third party logistics services, whereas the financial department considers 'money back guarantee' is far more critical in his business context. Consider another example within the QA 'on-time service', sales people concerns more about the 'on-time delivery' $\mathrm{AC}$, while trading people regards the 'on-time pickup' more important if, for instance, the cargo is perishable easily in their own warehouse.

\section{B. Service Provider Pair-wise Comparison}

This step involves determining how each service provider relatively supports the relevant Assessment Criteria. Suppose

two sets $S P=\left\{s p_{1}, \mathrm{~K}, s p_{n}\right\}$ and $C R=\left\{c r_{1}, \mathrm{~K}, c r_{n}\right\}$, where $S P$ represents all the candidate service providers and $C R$ corresponds to all the identified assessment criteria. Given any two service providers $s p_{i} \in S P$ and $s p_{j} \in S P$, we provide a pair-wise comparison $c o m p_{s p_{i} s p_{j}}$ under each Assessment Criterion $c r_{i} \in C R$. The comparison is based on a ratio scale with the numeric value, i.e. the "Scale of Measurement for AHP" [8]. In theory, any numbers less than infinity can be used for the upper bound. However, extensive practical experience [9] suggests that ' 9 ' is a good upper bound to use. Hence we use the scale presented in Table 2 to measure the comparative result. Further more, the ratio scale is reciprocal; i.e., $\operatorname{comp}_{s p_{i} s p_{j}}=1 /$ $c o m p_{s p_{j} s p_{i}}$ for all $s p_{i}, s p_{j} \in S P$. It is assumed in this paper that such a pair-wise comparison can be conducted in a semi-automatic, objective manner and based on multiple external information sources such as past experience, history record, or statistical forecasting, etc.

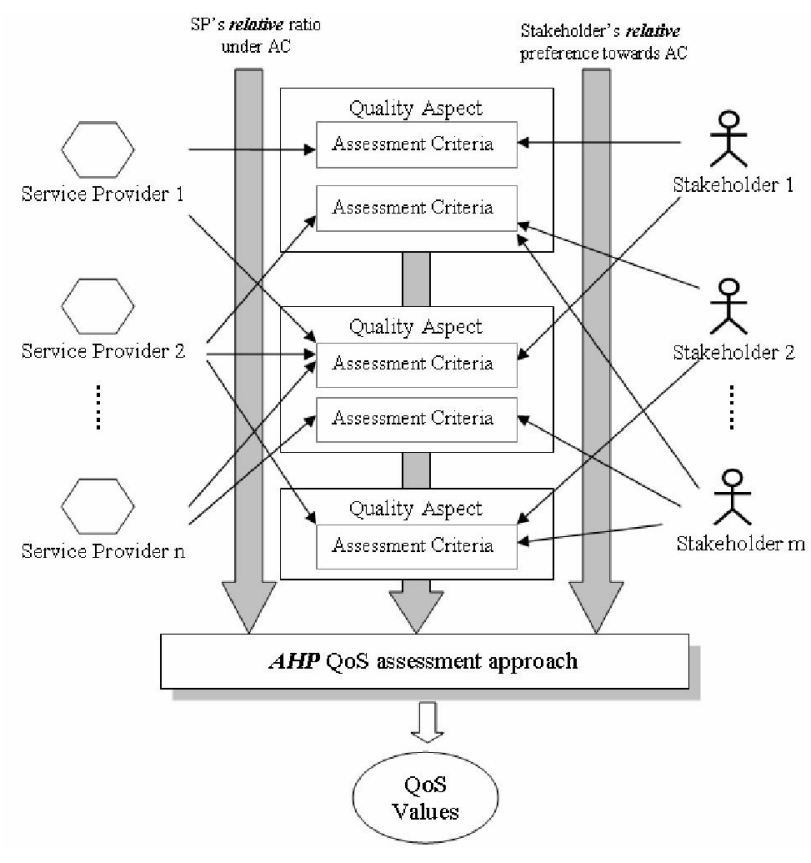

Figure 3. AHP QoS Assessment Approach

\section{Weighting Service Provider}

As a result of following the step in Section 3.2, for any given $c r_{t} \in C R$, we can have an $N \times N$ matrix $S_{C r_{t}}$, where $N$ is the total number of $s p_{i} \in S P$. For each matrix $S_{c r_{t}}$, we calculate the eigenvector to estimate the overall rankings of the service provider candidates from the pair-wise comparison conducted in 3.2. Thus given the matrix:

$$
S_{c r_{t}}=\left[\begin{array}{cccc}
c o m p_{s p_{1} s p_{1}} & \operatorname{comp}_{s p_{1} s p_{2}} & \ldots & \operatorname{comp}_{s p_{1} s p_{n}} \\
c o m p_{s p_{2} s p_{1}} & c o m p_{s p_{2} s p_{2}} & \ldots & c o m p_{s p_{2} s p_{n}} \\
. . & . . & \ldots & . . \\
c o m p_{s p_{n} s p_{1}} & c o m p_{s p_{n} s p_{2}} & \ldots & c o m p_{s p_{n} s p_{n}}
\end{array}\right]
$$

where $\operatorname{comp}_{s p_{i} s p_{i}}=1 / \operatorname{comp}_{s p_{j} s p_{i}}$ for all $\mathbf{i}, \mathbf{j}=1,2, \ldots, \mathbf{n}$

We use the eigenvector approach to derive a set of weights $W_{s p}=\left(w_{s p_{1}}, w_{s p_{2}}, \ldots w_{s p_{n}}\right)$ where $w_{s p_{i}} \in W$ is the weight that $s p_{i} \in S P$ under a criterion $c r_{t} \in C R$ so that the overall QoS weight can be formulated as follows:

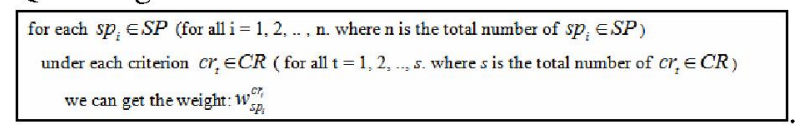

Table 2. Scale of Measurement for AHP

\begin{tabular}{|c|l|}
\hline $\begin{array}{c}\text { Numerical } \\
\text { Value }\end{array}$ & \multicolumn{1}{c|}{ Definition } \\
\hline 1 & Equally important or preferred \\
\hline 3 & Slightly more important or preferred \\
\hline 5 & Strongly more important or preferred \\
\hline 7 & Very strongly more important or preferred \\
\hline 9 & Extremely more important or preferred \\
\hline $2,4,6,8$ & Intermediate values to reflect compromise \\
\hline
\end{tabular}




\section{Weighting Quality Aspects}

The aim of this step is to determine the relative weight for every Quality Aspect (QA). The rationale behind this step is that the criteria should not always be considered equally weighted in the decision making. In particular, each QA has different importance that contributes to the ultimate QoS value; therefore, they should be assessed in a way that their respective significances are measured and integrated. Secondly, as indicated in Section 3.1 different stakeholders have different opinions on these preferences from their own perspectives. As a process of effective service provider selection, all their preferences should also be respected, and compromised if necessary. Suppose set $S H=\left\{s h_{1}, \mathrm{~K}, s h_{n}\right\}$, where $S H$ represents all the involved stakeholders in the process of service selection. Let set $Q A=\left\{q a_{1}, \mathrm{~K}, q a_{m}\right\}$, where QA represents all the Quality Aspects. Hence for each $s h_{i} \in S H$ (for all $\mathrm{i}=1,2$, ., $\mathrm{n}$. where $\mathrm{n}$ is the total number of $s h_{i} \in S H$ ), given any two $q a_{j}$ and $q a_{k} \in Q A$, we provide a pair-wise comparison $\operatorname{comp}_{q a_{j} q a_{k}}$ (for all $\mathbf{j}, \mathrm{k}=1,2, . ., \mathrm{m}$. where $\mathrm{m}$ is the total number of $q a_{k} \in Q A$ ). Such a comparison is the equivalent as in the Section 3.2 by repeating the steps in Section 3.2 , and 3.3 except that it is QA rather than SP are compared here. Thus, we can eventually obtain $w_{q a_{j}}^{s h_{i}}$ that represents the weight of each quality aspect $q a_{j} \in Q A$ in the view of a stakeholder $s h_{i} \in S H$. Next these $w_{q a_{j}}^{s h_{i}}$ are cumulated by employing the aggregation function so we can have $w_{q a_{j}}=\underset{i=1}{\operatorname{Ag} g r}\left(w_{q a_{j}}^{s h_{i}}\right)$. The aggregation function we choose here is the geometric mean as it is the distinctively suitable rule for combining preferences because it preserves the reciprocal property in the combined pair-wise comparison matrix in the AHP approach. Aggregating all stakeholders' preferences, we can thus formulate a set of QA weights as follows:

$W_{q a}=\left(w_{q a_{1}}, w_{q a_{2}}, \ldots w_{q a_{m}}\right)$

\section{E. Weighing Assessment Criteria}

In this step the relative weight of each Assessment Criterion (AC) is calculated. This weighting step looks into two types of weights: 1) the local weight refers to the relative priorities between ACs that are under the same QA. 2) the global weight is the relative priorities among all ACs in this QoS assessment, and is the $\mathrm{AC}$ weight that is used in producing the overall QoS value. Both weight calculations need aggregating the preferences from different stakeholders.

\section{Local AC Weighting}

Suppose that a $q a_{k} \in Q A$ has a set $C R_{q a_{k}}=\left\{c r_{1} \mathrm{~K}, c r_{o}\right\}$. And for each $s h_{i} \in S H$ (for all $\mathrm{i}$ $=1,2, . ., \mathrm{n}$. where $\mathrm{n}$ is the total number of $s h_{i} \in S H$ ), given any two $c r_{p}$ and $c r_{q} \in C R_{q a_{k}}$, we provide a pairwise comparison $\operatorname{comp}_{c r_{p} c r_{q}}$ (for all $\mathrm{p}, \mathrm{q}=1,2, \ldots, \mathrm{o}$. where $\mathrm{o}$ is the total number of all the ACs under $q a_{k} \in Q A$ ). Such a comparison is the equivalent as in the Section 3.4 except that it is AC rather than QA are compared here. Thus, we can eventually obtain $w_{c r_{p}}^{s h_{i}}$ that represents the weight of each assessment criterion $c r_{p} \in C R_{q a_{k}}$ under $q a_{k} \in Q A$ in the view of a stakeholder $s h_{i} \in S H$. Again, we then employ the geometric mean function to aggregate the preferences so that we can formulate a set of local (i.e. under $q a_{k} \in Q A$, AC weights as follows:

$L W_{c r}^{q a_{k}}=\left(w_{c r_{1}}, w_{c r_{2}}, \ldots w_{c r_{o}}\right)$

\section{Global AC Weighting}

Since for each $q a_{k} \in Q A$, we have the set $L W_{c r}^{q a_{k}}$, we can 'globalise' this set of weights by performing the following tasks:

1 Get the weight of $q a_{k}: w_{q a_{k}}$ from (1) in Section 3.4

2)Calculate global AC weighting $G W_{c r}^{q a_{k}}=w_{q a_{k}} \times L W_{c r}^{q a_{k}}$

Thus the final Global weighting for any $\mathrm{AC}$ can be formulated as follows:

$g w_{c r_{t}}^{q a_{k}}($ for all $1<=\mathrm{k}<=\mathrm{m}, 1<=\mathrm{t}<=0$ )

where $\mathrm{m}$ is the number of QA, $\mathrm{o}$ is the number of the members in the set $C R_{q a_{k}}$ under $q a_{k} \in Q A$ )

\section{F. The Composite QoS Weights}

After the above steps, we compute the composite QoS weight of the service providers $i$ in this service context by summing the weight under each $c r_{t} \in C R$ times the weight of each $c r_{t} \in C R$. This can be depicted as:

$$
W_{s p_{i}}=\sum_{q a_{k=1}}^{m} w_{s p_{i}}^{c r_{i}} g w_{c r_{i}}^{q a_{k}}
$$

\section{A CaSe STudy}

In this section, we provide a concrete example to illustrate the proposed quality assessment process. Let us first consider the following scenario:

Company ACME Ltd. has a freight waiting for being delivered from Beijing, China to Perth, WA and then to Sydney, NSW. This freight includes ten cartons of fragile antique articles including paintings, vases and glasses that are to be displayed in the WA Art Gallery and NSW Museum at two exhibitions on May 5 and May 10, 2006 respectively. 
Being unfamiliar with the Australian market at all, ACME seeks decisive advices from the consultancy AusLogiBroker Pty., asking for the most appropriate Australian third party logistics service provider to carry out such an international delivery service. The ACME thus submits to the AusLogiBroker Pty the following criteria in searching for the most suitable logistics service provider - SP:

The total willing-to-pay cost ranges from AU\$ 2500.00 to \$ 3500.00 , which includes packing, door to door delivery service from Beijing to Perth and to Sydney, GST, and shipping insurance.

- Packing and picking shall be on the same day. Thus the SP has to pack the fragile items and pick up the freight at ACME Ltd's office in Beijing by April 28, 2006.

- The freight has to reach WA Art Gallery without any loss at least one day before May 4.

- The freight has to reach NSW Museum without any loss at least one day before May 9.

- ACME Ltd can track and trace the status of the freight by using the online system provided by SP at anytime.

- The SP can issue signature-based proof for 'Undeniable Delivery' once the freight is acknowledged by authenticated receivers.

- If the freight is not delivered on time due to SP's own responsibilities, the $S P$ has to refund the freight costs.

Based on these initial criteria and the requirement specifications, AusLogiBroker has quickly found from its own database four potential Australian logistics providers that have past similar delivery records, and hence are capable of handling this freight delivery: Company $\mathrm{A}, \mathrm{B}, \mathrm{C}$, and D.

\section{A. Hierarchy Formation}

AusLogiBroker converts the decision making problem into the hierarchical structure. This is started by defining the Quality Aspect and Assessment Criterion based on the requirements captured from ACME. As shown in Figure 4, five major quality aspects offer the first level under the QoS context element: on time service, delivery, tracking capability, cost, and risk. The second level lists their associated assessment criteria. For example, 'on time service' aspect has two criteria to assess: 'on time pickup' and 'on time delivery'. Please note that for the sake of simplicity when calculating, we have only assigned one criterion for some QAs (e.g. delivery, cost, and tracking capability), however, more criteria can be easily appended as new requirements are proposed without changing the approach itself.

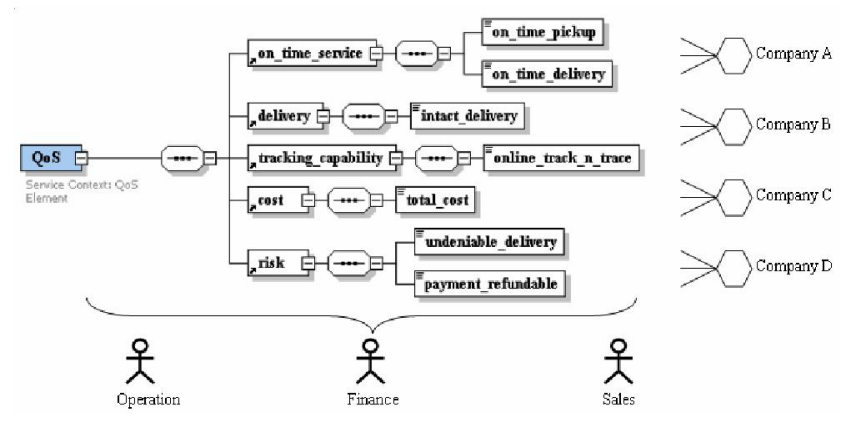

Figure 4. The Hierarchical Structure

Moreover, AusLogiBroker also identified three ACME departments as different stakeholders involved in selecting the logistics service providers: operation, finance, and sales team.

\section{B. Pair-wise Comparison}

In this step, we list the actual pair-wised comparison matrix for each service provider under each assessment criterion in Table $3-9$, the scale is based on Table 2 and then we use the eigenvector method proposed in [10] to estimate the relative weight.

\begin{tabular}{|c|c|c|c|c|c|}
\hline & \multicolumn{4}{|c|}{ Pair-wise Companison } & \\
\hline & Compary A & Company B & Compquary C & Company D & Relative Weight \\
\hline Compary A & 1 & $1 / 3$ & 2 & 5 & 0.259 \\
\hline Company B & 3 & 1 & 4 & 5 & 0.537 \\
\hline Compary C & $1 / 2$ & $1 / 4$ & 1 & 2 & 0.132 \\
\hline Commany D & $1 / 5$ & $1 / 5$ & $1 / 2$ & 1 & 0.072 \\
\hline
\end{tabular}

\begin{tabular}{|c|c|c|c|c|c|}
\hline & \multicolumn{4}{|c|}{ Pair-wise Companison } & \\
\hline & Conquary A & Company B & Conpayy C & Company D & Relative Weight \\
\hline Compary A & 1 & 5 & 1 & 4 & 0.421 \\
\hline Cormary B & $1 / 5$ & 1 & 2 & 2 & 0.246 \\
\hline Compary C & 1 & $1 / 2$ & 1 & 2 & 0.229 \\
\hline Company D & $1 / 4$ & $1 / 2$ & $1 / 2$ & 1 & 0.104 \\
\hline
\end{tabular}

Table 5. comparison matrix 'intact delivery"

\begin{tabular}{|c|c|c|c|c|c|}
\hline & \multicolumn{4}{|c|}{ Pair-wise Companison } & \\
\hline & Conpany A & Company $B$ & Conmany C & Convany D & Relative Weight \\
\hline Compary A & 1 & 2 & 5 & 6 & 0.538 \\
\hline Compary B & $1 / 2$ & 1 & 2 & 3 & 0.253 \\
\hline Compary C & $1 / 5$ & $1 / 2$ & 1 & 2 & 0.130 \\
\hline Conmary D & $1 / 6$ & $1 / 3$ & $1 / 2$ & 1 & 0.079 \\
\hline
\end{tabular}

Table 6. comparison matrix 'online track and trace'

\begin{tabular}{|l|c|c|c|c|c|}
\hline & \multicolumn{5}{|c|}{ Pair-mise Companison } \\
\hline & Conquary A & Compary B & Company C & Comparyy D & Relative Weight \\
\hline Company A & 1 & 1 & $1 / 7$ & $1 / 6$ & 0.063 \\
\hline Company B & 1 & 1 & $1 / 8$ & $1 / 7$ & 0.059 \\
\hline Company C & 7 & 8 & 1 & 2 & 0.530 \\
\hline Comparyy D & 6 & 7 & $1 / 2$ & 1 & 0.348 \\
\hline
\end{tabular}

Table 7. comparison matrix 'undeniable delivery"

\begin{tabular}{|l|c|c|c|c|c|}
\hline & \multicolumn{5}{|c|}{ Pair-mise Companison } \\
\hline & Congary A & Compary B & Compary C & Company D & Relative Weight \\
\hline Compary A & 1 & $1 / 2$ & 4 & 5 & 0.324 \\
\hline Compary B & 2 & 1 & 5 & 6 & 0.508 \\
\hline Conpary C & $1 / 4$ & $1 / 5$ & 1 & 2 & 0.103 \\
\hline Company D & $1 / 5$ & $1 / 6$ & $1 / 2$ & 1 & 0.066 \\
\hline
\end{tabular}

Table 8. comparison matrix 'payment refundable'

\begin{tabular}{|c|c|c|c|c|c|}
\hline & \multicolumn{4}{|c|}{ Pair-wise Companison } & \\
\hline & Compary A & Company B & Company C & Company D & Relative Weight \\
\hline Compary A & 1 & 3 & $1 / 2$ & 2 & 0318 \\
\hline Compary B & $1 / 3$ & 1 & $1 / 6$ & $1 / 2$ & 0.100 \\
\hline Compary C & 2 & 6 & 1 & 4 & 0.443 \\
\hline Compary D & $1 / 2$ & 2 & $1 / 4$ & 1 & 0.138 \\
\hline
\end{tabular}

Table 9. comparison matrix with respect to to tal cost'

\begin{tabular}{|l|c|c|c|c|c|}
\hline & \multicolumn{5}{|c|}{ Pair-wise Companison } \\
\hline & Conqany A & Company B & Conquary C & Compary D & Relative Weight \\
\hline Compary A & 1 & $1 / 9$ & $1 / 3$ & $1 / 4$ & 0.059 \\
\hline Compary B & 9 & 1 & 3 & 2 & 0.513 \\
\hline Compary C & 3 & $1 / 3$ & 1 & $1 / 2$ & 0.160 \\
\hline Compary D & 4 & $1 / 2$ & 2 & 1 & 0.269 \\
\hline
\end{tabular}




\section{Quality Aspect Preference}

Now for each stakeholder we collect their preference on each quality aspects. This generates another three comparison matrixes. For the page limit, we only list the comparison matrix of stakeholder Finance as shown in Table 10.

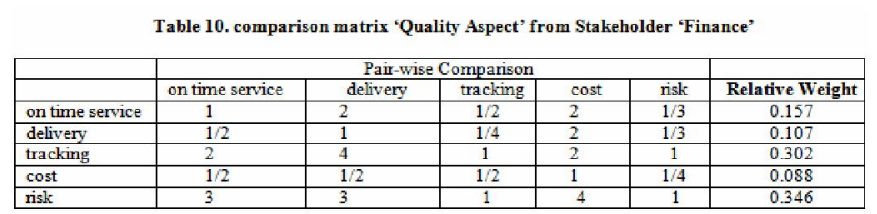

After gathering quality aspect preference for each stakeholder, different stakeholder preferences are then aggregated as described in Section 3.4 by employing the geometric mean function. This is illustrated in Table 11 .

\begin{tabular}{|l|c|c|c|c|}
\multicolumn{5}{c|}{ Table 11. Aggregated Quality Aspect Preferences } \\
\cline { 2 - 4 } Quality Aspects & Operation & Stakeholders & Aggregated \\
& Feighance & Sales & Weight \\
\hline on time service & 0.213 & 0.157 & 0.251 & 0.203 \\
\hline delivery & 0.371 & 0.107 & 0.183 & 0.194 \\
\hline tracking & 0.074 & 0.302 & 0.284 & 0.185 \\
\hline cost & 0.208 & 0.088 & 0.139 & 0.136 \\
\hline risk & 0.134 & 0.346 & 0.152 & 0.192 \\
\hline
\end{tabular}

\section{Assessment Criteria Preference}

First we need to calculate the local AC preference for each stakeholder. Note for intact delivery, tracking and cost, their local $\mathrm{AC}$ preference is 1 since only one criterion for each quality aspect and hence their global AC preference = QA preference. Hence we only need to compare and estimate the AC (both local and global) preferences for 'on time pickup, on time deliver, undeniable delivery, and payment refundable'. The final weight result for each AC under each stakeholder is recorded in Table 12.

Table 12. Aggregated Assessment Criteria Preferences
\begin{tabular}{|l|c|c|c|c|c|}
\hline \multicolumn{1}{|c|}{$\begin{array}{c}\text { Assessment } \\
\text { Criteria }\end{array}$} & \multicolumn{2}{|c|}{ Stakcholders (local AC preference) } & $\begin{array}{c}\text { Aggregrated } \\
\text { Lacal Weight }\end{array}$ & Global Weight \\
\cline { 2 - 5 } & Operation & Finance & Sales & \\
\hline on time pickup & 0.6 & 0.5 & 0.1 & 0.311 & 0.063 \\
\hline on time delivery & 0.4 & 0.5 & 0.9 & 0.565 & 0.115 \\
\hline intact delivery & 1 & 1 & 1 & 1 & 0.194 \\
\hline online track n trace & 1 & 1 & 1 & 1 & 0.185 \\
\hline cost & 1 & 1 & 1 & 1 & 0.136 \\
\hline undeniable delivery & 0.7 & 0.2 & 0.7 & 0.461 & 0.089 \\
\hline paymert refindable & 0.3 & 0.8 & 0.3 & 0.416 & 0.080 \\
\hline
\end{tabular}

C. Wu and E. Chang, "A Conceptual Architecture of Distributed Web Services for Service Ecosystems," presented at ISCA 18th International Conference on Computer Applications in Industry and Engineering, Honolulu, Hawaii, USA, 2005.

[2] J. C. Cimetiere, "Web Services Adoption and Technology Choices - Analysis of survey results," Technical Report, Group SQLI, TechMetrix Research, 2003.

[3] E. M. Maximilien and M. P. Singh, "Toward autonomic web services trust and selection," presented at 2nd International Conference of ServiceOriented Computing, New York, USA, 2004.

[4] D. J. Power, Decision Support Systems Hyperbook. Cedar Falls: IA: DSSResources.COM, 2000.

[5] E. Chang, T. S. Dillon, and H. K. Hussain, Trust and Reputation for Service-Oriented Environments: Technologies for Building Business Intelligence and Consumer Confidence: John Wiley \&

\section{E. Composite Weight Calculation}

Finally we can estimate each provider's relative weight under each quality assessment criterion by calculating the product of weight and global preference as shown in Table 12.

\begin{tabular}{|c|c|c|c|c|c|c|c|c|}
\hline \\
\hline & pickup & delivery & delivery & and trace & delivery & refundable & $\operatorname{cost}$ & Weight \\
\hline preferences & 0.063 & 0.115 & 0.194 & 0.185 & 0.136 & 0.089 & 0.080 & \\
\hline Company A & 0.259 & 0.421 & 0.538 & 0.063 & 0.324 & 0.318 & 0.059 & 0.258 \\
\hline Compary B & 0.537 & 0.246 & 0.253 & 0.059 & 0.508 & 0.100 & 0.513 & 0.241 \\
\hline Conpany C & 0.132 & 0.229 & 0.130 & 0.530 & 0.103 & 0.443 & 0.160 & 0.224 \\
\hline Company D & 0.072 & 0.104 & 0.079 & 0.348 & 0.066 & 0.138 & 0.269 & 0.139 \\
\hline
\end{tabular}

\section{Conclusion}

In this paper, we proposed a new solution for QoS-based services selection. Our solution is based on our observation that the service selection can be deemed as a process of decision making. Hence, the solution makes decisions for selecting the most suitable services by leveraging a DSS module - AHP Model, which relies on measuring the weight against certain Quality Criteria defined in Context-specific Quality Aspects. To realise our solution, we provide DSS module design. Currently, proof-of-concept prototyping work is ongoing. For the future work, we will focus on the implementation of the DSS module prototype embedded in our distributed web services architecture, upon which the service ecosystem is built. Furthermore, the AHP Model based QoS selection simulation is also need to be carried out and the effectiveness measurement metrics should be formulated in a formative way. In particular, the selflearning and recommendation mechanisms in terms of the pair-wise comparison in DSS module is a very interesting and promising direction for our future work.

\section{REFERENCES}

Sons, 2005.

[6] T. L. Saaty, The Analytic Hierarchy Process: McGraw-Hill, New York, 1980.

[7] E. H. Forman and M. A. Selly, Decision By Objectives - how to convince others that you are right: World Scientific Publishing Co. Pte. Ltd., 2001.

[8] B. L. Golden, E. A. Wasil, and P. T. Harker, The Analytic Hierarchy Process - Applications and Studies: Springer-Verlag, 1989.

[9] P. T. Harker and L. G. Vargas, "Theory of Ratio Scale Estimation: Saaty's Analytic Hierarchy Process," Management Science, vol. 33, pp. 1383 1403, 1987.

[10] T. L. Saaty and J. Alexander, Thinking with Models: Pergamon Press, Oxford, England, 1981. 